\title{
Impacts écologiques et socio-économiques de Ceratophyllum demersum L., une plante aquatique envahissante dans le delta du fleuve Sénégal
}

\author{
Sanou NDOUR ${ }^{1 *}$, Mame Samba MBAYE ${ }^{1}$, Jules DIOUF ${ }^{1}$, Rahimi MBALLO ${ }^{1}$, Maniane \\ SARR $^{1}$, Mallé GUEYE ${ }^{2}$ et Kandioura NOBA ${ }^{1}$ \\ ${ }^{I}$ Laboratoire de Botanique et Biodiversité, Département de Biologie Végétale, Faculté des Sciences et \\ Technique, Université Cheikh Anta Diop, B.P. 5005 Dakar-Fann, Sénégal. \\ ${ }^{2}$ Direction des Parcs Nationaux, Ministère de l'Environnement et du Développement Durable, B.P 5135 , \\ Dakar-Fann, Sénégal. \\ *Auteur correspondant ; E-mail : colettsanou@gmail.com/Tel : +221 772556736.
}

\section{RESUME}

Les zones humides sont des ressources vitales riches en biodiversité. Elles procurent des biens et services, et contribuent à l'atténuation des changements climatiques. Cependant, au Sénégal, ces écosystèmes subissent un phénomène alarmant de dégradation du fait des effets combinés de plusieurs facteurs dont $C$. demersum, une espèce exotique envahissante rencontrée dans les eaux du fleuve Sénégal. Cette présente étude a pour objectif d'évaluer les impacts écologiques et socio-économiques liés à l'envahissement de $C$. demersum dans le delta du fleuve Sénégal. L'étude a consisté à réaliser des enquêtes et des entretiens auprès des populations des sept (7) villages périphériques du Parc National des Oiseaux du Djoudj et des agents de la Société d'Aménagement et d'Exploitation des terres du delta (SAED). Les résultats de l'étude ont montré que la grande majorité (98\%) de la population est constituée d'agriculteurs, la prolifération de l'espèce est récente et remonte à la mise en service des barrages de Diama et de Manantali. Le comblement des plans d'eau, la baisse du rendement de pêche et l'apparition des maladies dites hydriques sont les nuisances qui impactent le plus l'écosystème du delta du fleuve Sénégal et la vie des communautés. Ces résultats obtenus sont importants dans la conservation de cette zone humide à importance internationale et la mise en place d'un dispositif de gestion écologique pour la restauration du milieu.

(C) 2019 International Formulae Group. All rights reserved.

Mots clés : Impact, espèce invasive, zone humide, Fleuve Sénégal.

\section{Ecological and socio-economic impacts of Ceratophyllum demersum L., an invasive aquatic plant in the Senegal River delta}

\begin{abstract}
Wetlands are vital resources rich in biodiversity. They provide goods and services, and help mitigate climate change. However, in Senegal, these ecosystems are experiencing an alarming degradation phenomenon due to the combined effects of several factors including $C$. demersum, an invasive alien species encountered in the waters of the Senegal River. This study aims to assess the ecological and socio-economic impacts of $C$. demersum invasion in the Senegal River delta. The study consisted in carrying out surveys and interviews with
\end{abstract}


the populations of the seven (7) outlying villages of the Djoudj Bird National Park and agents of the Society for Development and Exploitation of the Delta Lands (SAED). The results of the study showed that the vast majority $(98 \%)$ of the population consists of farmers, the proliferation of the species is recent and dates back to the commissioning of Diama and Manantali dams. The filling of water bodies, the fall in fishing yield and the appearance of so-called water-related diseases are the most damaging impacts on the ecosystem of the Senegal River delta and the life of the communities. These results are important in the conservation of this wetland of international importance and the establishment of an ecological management system for the restoration of the environment.

(C) 2019 International Formulae Group. All rights reserved.

Keywords: Impact, invasive species, wetland, Senegal River.

\section{INTRODUCTION}

L'impact des espèces exotiques envahissantes sur les écosystèmes, les habitats et les espèces indigènes sont graves et souvent irréversibles. Ces espèces invasives s'établissent et répandent leurs menaces sur des écosystèmes et des habitats variés (Gueye et Noba, 2016). Elles sont considérées comme une des principales causes d'érosion de la biodiversité au niveau mondiale (UICN, 2000; Mack et al., 2000). Ces phénomènes sont particulièrement accrus dans les îles océaniques où elles sont perçues comme le premier facteur d'extinction d'espèces et de transformation des écosystèmes (Donlan et Wilcox, 2008; Sax et Gaines, 2008). La perte de la biodiversité et l'altération du fonctionnement des écosystèmes sont les dommages les plus fréquemment associés aux espèces invasives (Gueye et al., 2016). Or, si en théorie la lutte contre les espèces exotiques envahissantes concerne en priorité leurs impacts sur la biodiversité et sur les écosystèmes, bien souvent les interventions de terrain ont lieu lorsque celles-ci ont un impact sur les usages du milieu (GISD, 2006). Ces usages sont liés le plus souvent aux différents secteurs dans lesquels s'activent les populations riveraines des zones envahies. Ces impacts peuvent être enregistrés dans les domaines de l'agriculture, de l'élevage, de la pêche, du tourisme et dans le domaine de la santé des usagers.

Les dommages causés par les espèces invasives à l'échelle globale sont estimés à plus de 1400 milliards de dollars, l'équivalent de $5 \%$ de l'économie mondiale (Pimentel et al., 2001; The World Bank, 2014). Le coût économique de leur gestion se chiffre en milliards d'euros dans le monde. En Europe, une première estimation situe leur coût entre 9 et 12 milliard d'euros par an (Shine et al., 2009). Des études aux Etats-Unis et en Inde ont montré que le coût économique s'élève à 1300 milliards de dollars US/an (GISD, 2006).

Sur le plan de la santé publique, ces espèces peuvent être allergènes (Pomerleau, 2017) ou constituer des réservoirs (Diouf et al., 2019) et/ou des vecteurs de maladies pour l'homme et le bétail. Ainsi, la prise en compte des nuisances sur l'économie (activités humaines) et le bien-être humain (santé publique et activités de loisir) que provoquent ces espèces parait aujourd'hui comme une préoccupation majeure dans le delta du fleuve Sénégal qui connait une prolifération spectaculaire de certaines espèces végétales comme C. demersum, une macrophyte aquatique envahissante dans la zone.

C. demersum a été répandue dans le monde entier par l'intermédiaire du commerce de l'aquarium et de l'étang. Elle est considérée comme une mauvaise herbe des cours d'eau dans de nombreuses régions du monde, en raison de sa capacité à se propager rapidement, à envahir de larges superficies d'habitats aquatiques et à croître à des profondeurs plus élevées que d'autres espèces de mauvaises herbes (GISD, 2006; Cottaz, 2016).

C'est dans ce contexte que cette étude a été initiée afin d'évaluer les impacts écologiques et socio-économiques liés à l'envahissement de cette espèce sur les populations des villages périphériques du Parc National des Oiseaux du Djoudj et leur activités. Il s'agit plus particulièrement: de comprendre l'historique d'invasion de 
l'espèce, de déterminer les impacts sur le milieu, la santé des populations et les activités génératrices de revenus, mais aussi les stratégies de gestion utilisées par les riverains.

\section{MATERIEL ET METHODES}

\section{Présentation de la zone d'étude}

L'étude a été menée au niveau des villages périphériques du Parc National des Oiseaux du Djoudj que sont: Diadiam 1, Diadiam 2, Diadiam 3, Débi, Tiguet, Fourarad et Rhone (Figure 1). Classé depuis 1977 comme zone humide d'importance internationale par la convention Ramsar et inscrit sur la liste des sites du patrimoine mondiale en 1980, le Parc National des Oiseaux du Djoudj est considéré comme étant l'un des trois sanctuaires d'Afrique occidentale pour les oiseaux migrateurs mais également l'un des principaux sites de convergence des espèces d'oiseaux paléarctiques et afro tropicales (Treca, 1989 in Noba et al., 2010). Le parc joue ainsi un rôle important dans le processus de reproduction et d'hivernage pour plusieurs espèces d'oiseaux aquatiques mais également contribue à la création des emplois verts et au développement socio-économique de cette zone par des activités génératrices de revenus. Le choix du site d'étude se justifie non seulement par l'absence de données scientifiques portant sur les impacts des espèces invasives et plus particulièrement de C. demersum dont la prolifération prend des proportions inquiétantes dans cette zone humide d'importance internationale mais également par la spécificité des villages par rapport à leur relation avec les plans d'eau. En effet, les différentes activités des riverains sont étroitement liées aux cours d'eau soit dans l'approvisionnement en eau de boisson soit dans les secteurs d'activité économique.

Le parc est situé à près de 15 kilomètres de Ross-Béthio et 60 kilomètres au Nord-Est de Saint- Louis, dans le delta du fleuve Sénégal. Le climat est de type sahélien avec une alternance de deux saisons (pluvieuse et sèche) marqué par l'harmathan (Fall et al., 2003). La pluviométrie annuelle est faible et n'excède pas $300 \mathrm{~mm}$. La température moyenne annuelle est de $27{ }^{\circ} \mathrm{C}$ (Gueye, 2009). La végétation de type sahélien reflète le niveau relativement faible des précipitations sur des sols allomorphes défavorables avec des peuplements d'espèces épineuses comme les Acacia au niveau des zones inondées, des Tamarix au niveau des zones où la salinité est élevé et des Phragmites dans les berges et les digues (Fall et al., 2004).

\section{Collecte des données}

Les enquêtes semi structurées ont été réalisées par l'administration d'une fiche ou questionnaire établie avec le logiciel Sphinx. Les questions sont ouvertes et/ou fermées. Les informations collectées sont entre autre le secteur d'activité socioéconomique de l'enquêté, l'historique de l'invasion, les nuisances engendrées par la prolifération de cette espèce dans la zone d'étude et les stratégies de gestion utilisées par les usagers du milieu.

Un échantillonnage aléatoire simple a été appliqué. Pour ce faire, $5 \%$ des carrés de chaque village ont été interrogés (Satin et Shastry, 1983). Les personnes enquêtées étaient constituées d'hommes et de femmes dont l'âge était supérieur ou égal à 17 ans. Ce qui fait un total de 145 questionnaires répartis comme suit: Diadieum 1 (22), Diadieum 2 (19), Diadieum 3 (20), Débi, (23) Tiguet (26), Fourarate (13), Rhone (22).

\section{Traitement des données}

Le dépouillement et le traitement des données ont été faits sur Sphinx Plus et le tableur Excel. Les informations obtenues ont servi à la détermination des caractéristiques socioéconomiques des enquêtés. L'âge moyen des enquêtés, la fréquence de citation de chaque type de nuisance, les stratégies de gestion ainsi que le coût de la gestion ont été déterminés. Ainsi, les nuisances citées par les personnes ont été classées dans les quatre paramètres proposés par le protocole ISSIA que sont : 1) les impacts économiques (coûts de lutte contre l'espèce exotique envahissante) ;2) les impacts sanitaires sur les populations humaines ; 3 ) les impacts négatifs sur les activités humaines; 4) les impacts négatifs sur les activités de loisirs. Ce protocole nommé ISSIA (Invasive Species Socioeconomic (and Sanitary) Impact Assessment) est une méthode exclusivement basée sur l'évaluation des impacts socioéconomiques (et sanitaires) des espèces exotiques envahissantes (Cottaz, 2016). 


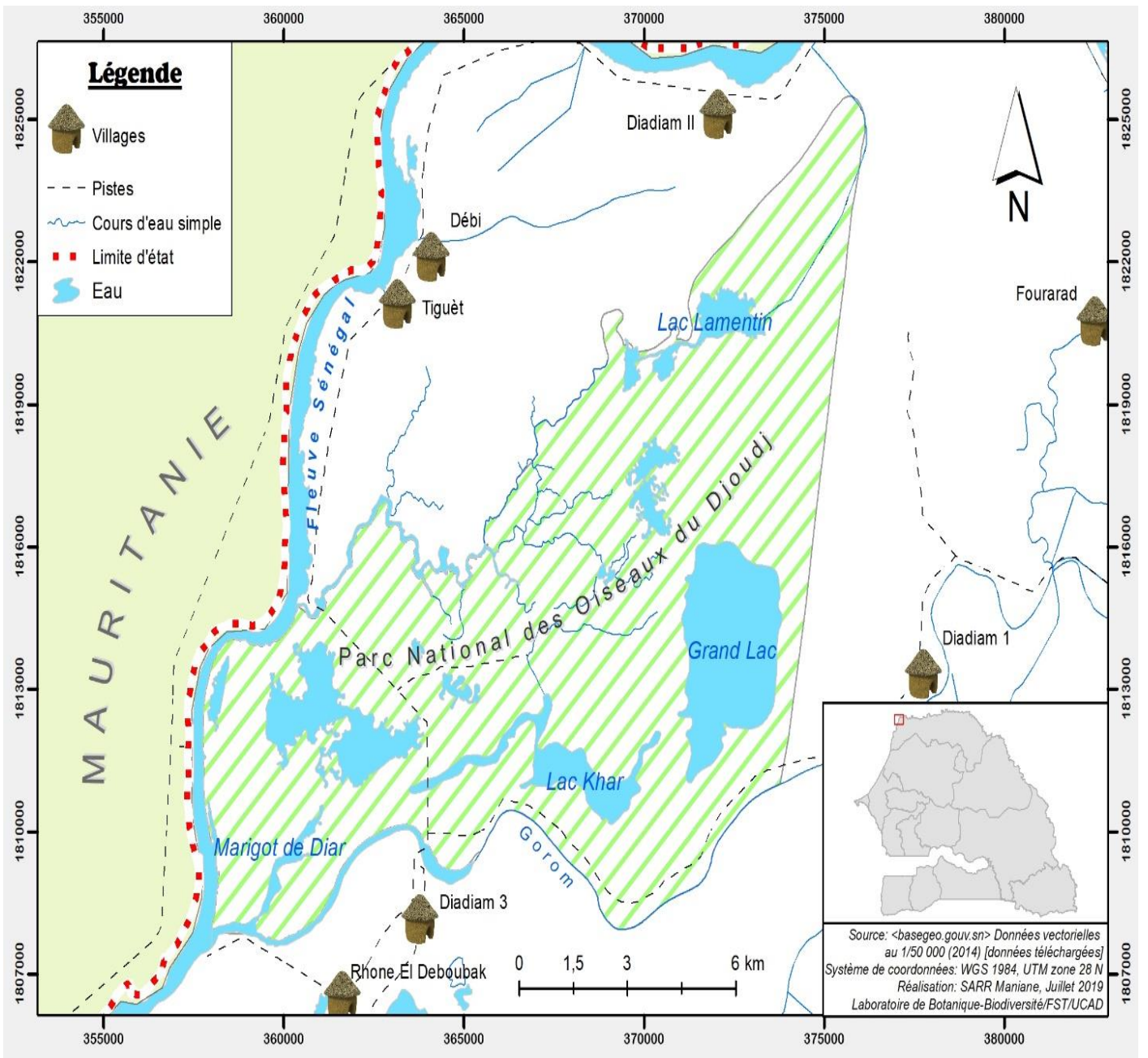

Figure 1: Carte de la zone d'étude.

\section{RESULTATS}

\section{Caractéristiques socioéconomiques des enquêtés}

$\mathrm{Au}$ total 145 personnes ont été interrogées. L'âge des enquêtés est compris entre 17 et 80 ans avec une moyenne de 44 ans. Ces enquêtés sont regroupés dans plusieurs catégories socioprofessionnelles dont les plus représentés sont les agriculteurs avec un effectif de 78 personnes, soit $53 \%$ des enquêtés, suivis des ménagères $(25,5 \%)$, des pêcheurs $(20,7 \%)$ et des éleveurs $(14,5 \%)$ (Figure 2). Pour ce qui est du genre, les femmes sont plus nombreuses que les hommes interrogés avec un pourcentage de $50,3 \%$ des personnes enquêtées.

\section{Impacts des espèces envahissantes Historique de l'invasion}

Quatre-vingt-dix-huit pourcent $(98 \%)$ des enquêtés ont affirmé que la prolifération de l'espèce était récente et quelle remonterait à la mise en service des barrages de Diama et de Manantali (Figure 3).

\section{Impact sur le milieu}

Trois nuisance majeures ont été signalées par les enquêtés en ce qui concerne l'impact sur le milieu (Figure 4). Le comblement des plans d'eau a été le plus cité $(73,1 \%)$ suivi de la destruction de l'habitat pour les poissons $(13,1 \%)$ puis de la perte de biodiversité $(4,1 \%)$. 


\section{Impacts sur la santé humaine}

Les nuisances sur la santé publique concernent essentiellement l'apparition et la recrudescence de maladies dites hydriques $(71,7 \%)$ comme la bilharziose, la schistosomiase, les diarrhées, les allergies (causées par les piqures d'une sangsue vivant sur $C$. demersum), les maux de ventre qui surviennent en cas de baisse du niveau de l'eau, et de l'altération de la qualité de l'eau qui devient impropre et dangereuse à la consommation $(57,9 \%)$ (Figure 5).

\section{Impacts sociétaux}

Deux impacts ont été mentionnés par les enquetés que sont la gêne dans les activités nautiques avec une fréquence de citation de $51,7 \%$ suivie de la diminution de la valeur esthétique du site $(13,1 \%)$ (Figure 6).

Impacts sur les activités génératrices de revenus

Dans la catégorie de l'agriculture, l'encombrement des ouvrages d'irrigation représentent $58,6 \%$ des citations, soit un effectif de 85 personnes sur les 144 enquêtées, suivi de la réduction de la vitesse d'écoulement de l'eau au niveau des axes hydrauliques $(43,5 \%)$ (Tableau 1$)$. La limitation des zones navigables constitue

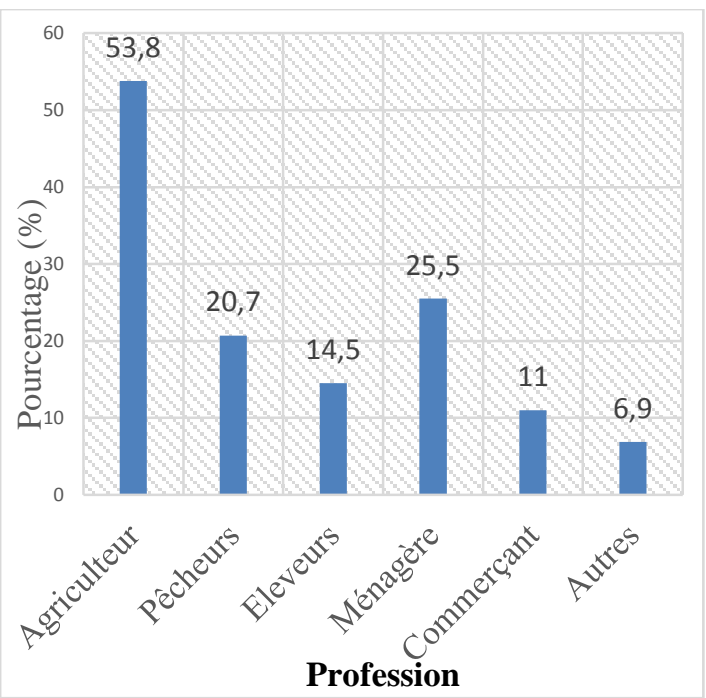

Figure 2: Catégories socio-professionnelles des enquêtés.
59,3\% des citations dans la catégorie de la pêche suivie de la baisse du volume de prise ou rendement de pêche $(57,2 \%)$, de la mortalité des poissons $(17,2 \%)$. En ce qui concerne la catégorie de l'élevage, l'apparition de maladies du bétail (douve du foie) représente $43,5 \%$, soit 63 citations, et la limitation de l'accès du bétail à l'eau (26,2\%).

Impacts économiques et stratégies de gestion utilisées par les usagers

L'arrachage mécanique $(59,31 \%)$ est la stratégie de gestion la plus utilisée par les riverains suivie de l'arrachage manuel $(40,61 \%)$ (Tableau 2). Selon le chef de Division et Maintenance des Réseaux de la SAED, ce coût est estimé à $100000 \mathrm{~F} \mathrm{CFA/h}$ pour l'utilisation de la pelle d'eau et à 80000 F CFA/h pour l'utilisation de la pelle à bras long. Cette technique d'enlèvement mécanique est utilisée au moins 8 heures par jour pendant 25 jours pour une campagne agricole de 4 mois. Certains petits exploitants ont recourt à l'arrachage manuel jugé moins coûteux. Elle consiste à employer une main d'œuvre moyennant 5000 F CFA la journée de travail. En effet, pour cette dernière, elle est jugée trop pénible avec des risques d'accident de travail élevés (morsure de serpent, noyade).

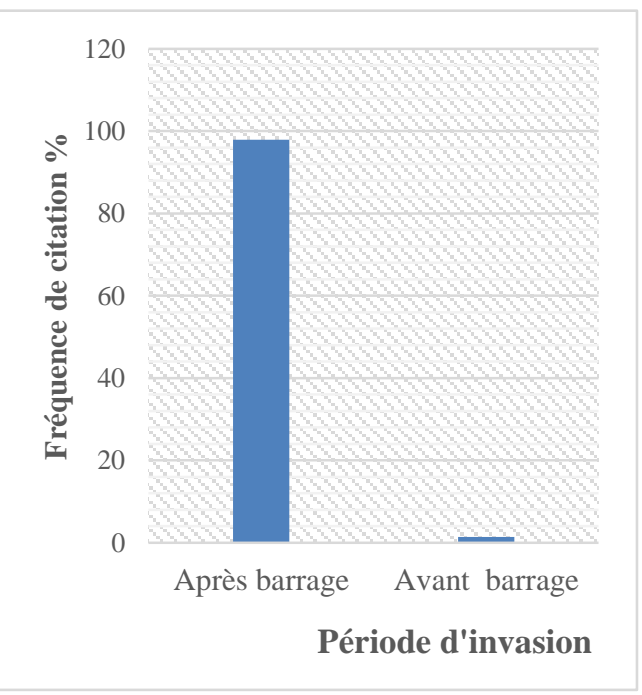

Figure 3: Historique de l'invasion de l'espèce. 


\section{Fréquence de citation}

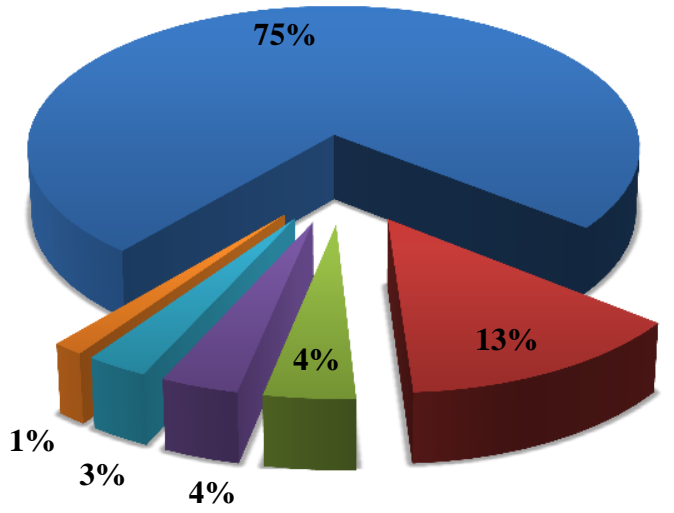

- Comblement des plans d'eau

— Dégradatsion de l'habitats des poissons

- Réduction de la biodiversité

घutrophisation du milieu

Envasement

— Mauvaise odeur de l'eau

Figure 4 : Impacts de la prolifération de l'espèce sur le milieu.

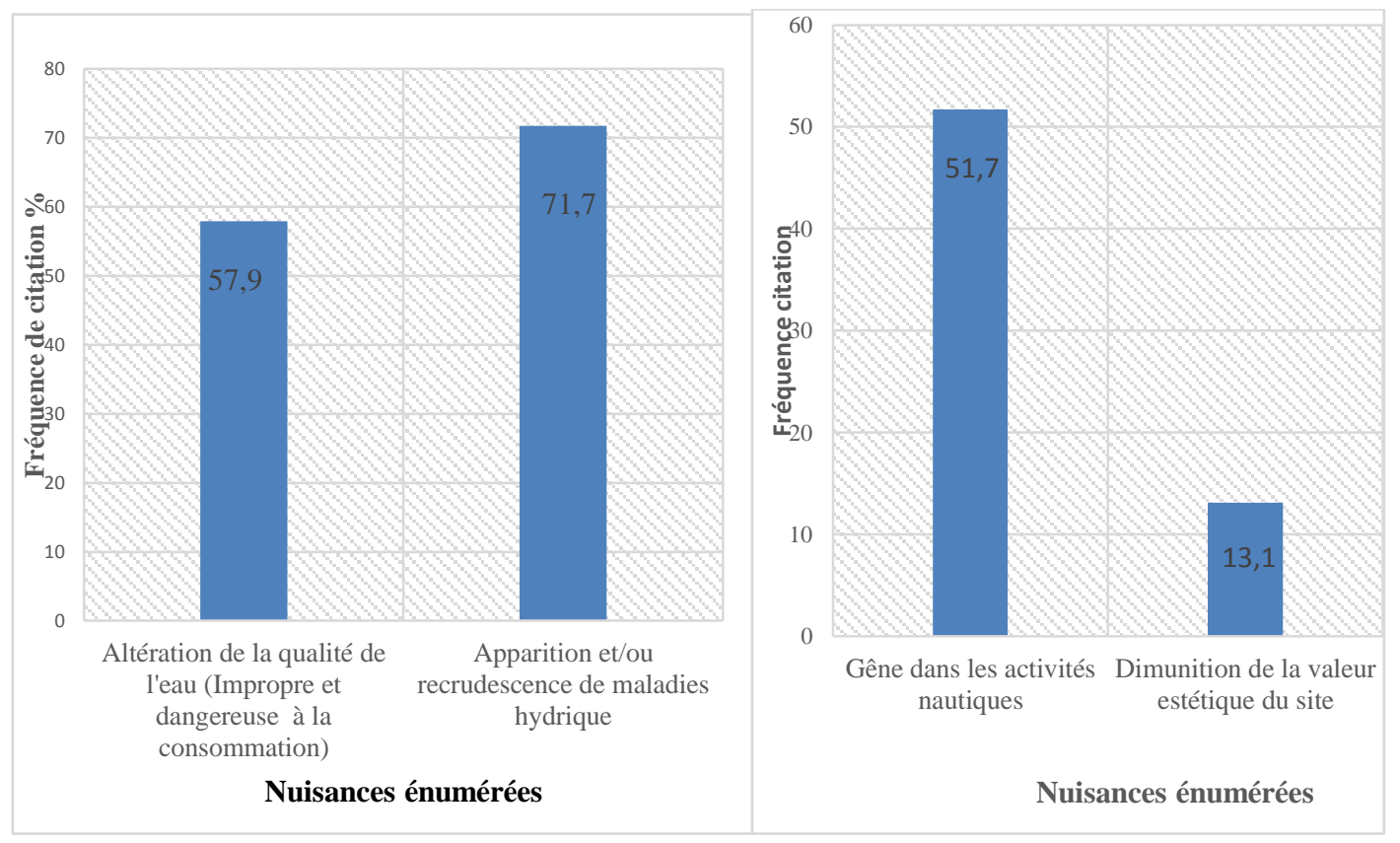

Figure 5 : Nuisances sur la santé humaine.

Figure 6 : Nuisances sur les activités de loisir. 
Tableau 1 : Nuisances sur les activités génératrices de revenus des populations.

\begin{tabular}{clc}
\hline & \multicolumn{1}{c}{ Impacts } & Fréquence de citation (\%) \\
\hline \multirow{2}{*}{ Pêche } & Limitation des zones navigables & 59,3 \\
& Baisse du rendement de pêche & 57,2 \\
& Mortalité des poissons & 17,2 \\
\hline \multirow{2}{*}{ Agriculture } & Encombrement des ouvrages d'irrigation & 58,6 \\
& Réduction de l'hydraulicité des axes & 43,5 \\
\hline \multirow{2}{*}{ Elevage } & Limitation de l'accès du bétail à l'eau & 26,2 \\
& Apparition de maladie chez le bétail (Douve du & 43,5 \\
& foie) & \\
\hline
\end{tabular}

Tableau 2 : Méthodes de gestion utilisées et leur cout moyen.

\begin{tabular}{ccccc}
\hline Méthodes de gestion & Nombre de citation & Proportion\% & Coût moyen riverain & Cout moyen SAED \\
\hline Arrachage manuel & 59 & 40,69 & $5000 \mathrm{FCFA} /$ jour & \\
\hline Arrachage mécanique & 86 & 59,31 & $55000 \mathrm{FCFA} / \mathrm{h}$ & $90.000 \mathrm{FCFA}$ \\
\hline
\end{tabular}

\section{DISCUSSION}

L'activité dominante exercée par les personnes interrogées lors de cette étude est l'agriculture. Ces résultats sont similaires à ceux de Bissoue et al. (2017) au niveau du fleuve Nyong dans la commune de Mbalmayo au Cameroun où l'activité dominante des acteurs est l'agriculture.

L'historique d'invasion de l'espèce remonte à la mise en service des barrages de Diama et Manantali. Néanmoins, cette espèce était présente dans l'écosystème du delta (Trochain, 1940) bien avant l'installation de ces aménagements hydroagricoles. Son potentiel de propagation était régulé par le régime naturel hydrologique du fleuve qui était marqué par l'alternance d'une période de crue avec des eaux douces abondantes et d'une période d'étiage avec une salinisation importante des sols et des eaux (Triplet et al., 2014). Le système ainsi artificialisé se traduit par la régularisation de la crue, le soutien à l'étiage durant la saison sèche, le contrôle des écoulements dans les terres du Delta et leur protection contre les intrusions de la langue salée (DPN, 2017)

L'apparition ou la recrudescence de maladies dites hydriques comme la bilharziose, la schistosomiase, la diarrhée, les maux de ventre dans le cas d'une baisse du niveau de l'eau est la nuisance qui affecte le plus le secteur de la santé des populations. Ce résultat pourrait s'expliquer par le fait que les riverains sont en contact permanent avec les eaux du fleuve pour les besoins d'approvisionnement en eau de boisson et/ou de baignade ce qui les expose aux risques de contamination par les vecteurs responsable de ces maladies (Poda et al., 2004; Zongo et al., 2017). Il existe une forte corrélation entre la densité des plantes aquatiques envahissantes dont $C$. demersum dans le delta du fleuve Sénégal et la transmission des cercaires responsables de la schistosomiase. En effet, Bolinus truncatus rohlfsi, mollusque hôte intermédiaire de Schistosoma haematobium (responsable de la schistosomiase) manifeste, pour son habitat, une préférence à $C$. demersum (Ndione et al., 2018). L'abondance des effectifs de $B$. rohlfsi est généralement associée à la présence dans leur biotope de végétaux aquatiques (Sarr et al., 2011; Gbocho et al., 2015).

Le comblement des plans d'eau suivi de la destruction de l'habitat des poissons, engendrant une perte de la biodiversité sont 
les principales menaces qui pèsent sur le parc et son environnement. Ces comblements sont à l'origine de la diminution des activités nautiques à cause des risques de noyade. Le myriophylle à épi, Myriophyllum spicatum, une espèce présentant le même type de colonisation que $C$. demersum, forme des herbiers monospécifiques créant d'épais tapis denses dans la zone littorale des lacs du sud du Québec (Pomerleau, 2017). Ces colonies peuvent entraver ou même rendre impossible la pratique d'activités telles que la baignade, la navigation de plaisance (Michon, 2015). Ces résultats sont similaires à ceux de Bissoue et al. (2017) qui ont montré que l'envahissement du lit des cours d'eau par les plantes invasives entraine une perte de biodiversité du milieu et empêche les activités de course de pirogue dans les sites envahis par les macrophytes.

Les impacts négatifs sur la pêche se soldent principalement par une baisse du volume de prise (rendement de pêche). $C$. demersum, en raison de sa capacité à former des lits monospécifiques denses ou forêts d'herbiers sous-marins pouvant aller jusqu'à $10 \mathrm{~m}$ de haut (Vári, 2012), provoque une diminution de la superficie des cours d'eau, une obstruction des voies de navigation et une perte de l'habitat essentiel pour les espèces de poisson et d'autres espèces animales d'eau douce importantes (NIWA, 2005). L'effet combiné de ces trois nuisances précédemment énumérées contribue activement à la réduction des volumes de prise des pêcheurs. En effet, l'encombrement réduit la superficie des cours d'eau, empêche les pêcheurs d'accéder à certaines zones qui étaient des endroits privilégiés de par leur richesse ictyofaunique et limite ainsi l'accès du bétail aux points d'eau.

A cela s'ajoute la perte de l'habitat entrainant la mortalité et la disparition de certaines espèces de poisson qui participe également à la réduction du volume de prise. La mort des plantes invasives dans les cours d'eau entraine un processus de sédimentation à l'origine de l'eutrophisation qui raréfie la quantité d'oxygènes dissous, rendant le milieu asphyxique et provoquant ainsi la mort des espèces animales du fleuve (Priso et al.,
2012). Une autre nuisance très perceptible est l'apparition de la douve du foie chez le bétail à la consommation de ces eaux. Cet impact n'a pas été évoqué dans le fleuve Nyong au Cameroun (Bissoue et al., 2017).

Ces phénomènes de comblement des plans d'eau peuvent entraver également le développement du phytoplancton de trois façons: par la concurrence à l'azote inorganique, par la compétition à la lumière et par l'allélopathie (GISD, 2006). Ce phytoplancton est indispensable à la reproduction et à la croissance des alevins pour le peuplement de ces milieux en poisson. Etant un maillon indispensable de la chaîne trophique, les algues jouent un rôle de base dans les écosystèmes aquatiques (Dibong et Ndjouondo, 2014). Ces résultats sont similaires à ceux de Bissoue et al., (2017) qui ont montré que l'envahissement des zones de fraie abritant des espèces de poisson strictement herbivores par les espèces végétales invasives réduit la biodiversité des sites et par conséquent induit à une baisse des rendements de pêche.

Sur l'agriculture, l'encombrement des ouvrages d'irrigation et la réduction de l'hydraulicité des axes de drainage sont les problèmes qui ont été imputés à la prolifération de $C$. demersum. L'obstruction des voies d'eau et des canaux d'irrigation rendent difficile voire impossible le drainage des superficies de culture. Ce résultat peut s'expliquer par le fait que l'espèce par sa capacité à former des herbiers denses le long des plans d'eau entraine une diminution de la vitesse d'écoulement ou débit des cours d'eau rendant ainsi difficile l'irrigation des terres de culture.

La méthode de gestion mécanique est la technique la plus utilisée par les usagers du milieu. Ces résultats sont similaire à ceux obtenus dans le fleuve Nyong au Cameroun (Bissoue et al., 2017). Par contre, ces résultats sont différents de ceux trouvés dans la même zone d'étude où la gestion manuelle est la plus répandue (Mbaye, 2015). Le recourt de plus en plus à la gestion mécanique pourrait être lié à plusieurs raisons dont les risques liés aux accidents de travail (morsure de serpents, noyade) mais également du fait que 
l'arrachage manuel est jugé trop pénible et moins efficace et efficiente. A cela s'ajoute le coût substantiel lié à l'enlèvement de la plante (Ndour, 2015) à l'entretien et à la maintenance de ces ouvrages d'irrigation.

\section{Conclusion}

Cette présente étude a permis de déterminer les impacts écologiques et socioéconomiques liés à l'invasion de $C$. demersum au niveau des 7 villages périphériques du Parc National des Oiseaux du Djoudj. Les résultats obtenus montre que $C$. demersum a une forte influence sur le milieu par le comblement des plans d'eau à près de $70 \%$. L'encombrement des ouvrages de drainage ou canaux d'irrigation $(58,7)$, la baisse du rendement de pêche $(57,2 \%)$ et l'apparition de la douve du foie chez le bétail (43,5\%) figurent respectivement parmi les nuisances qui affectent le plus l'agriculture, la pêche et l'élevage, principales secteurs d'activité des populations riveraines. Les usagers du site ont plus recourt à le l'arrachage mécanique $(59,31 \%)$ pour l'enlèvement de la plante. L'invasion de cette espèce menace les services écosystémiques qu'offre le delta du fleuve Sénégal. Son éradication par l'utilisation de méthodes de gestion efficaces, efficientes et durables est nécessaire pour le maintien du potentiel de l'agriculture irriguée, de la pêche et la préservation des eaux du fleuve et de sa biodiversité. Des techniques de gestion basées sur les méthodes de lutte biologique ainsi que sur la manipulation de l'habitat par l'utilisation de l'hydrodynamique du delta (lâchée d'eau de mer dans les cours d'eau) sont à envisager pour le contrôle de la propagation de l'espèce.

\section{CONFLIT D'INTERETS}

Les auteurs déclarent qu'ils n'y a aucun conflit d'intérêts pour cet article.

\section{CONTRIBUTIONS DES AUTEURS}

SN a collecté, traité et analysé les données puis rédigé l'article; MSM a confectionné la carte de la zone d'étude, JD, $\mathrm{RM}, \mathrm{MS}, \mathrm{KN}$ et MG ont contribué à la correction de cet article.

\section{REMERCIEMENTS}

Les auteurs de ce travail remercient le personnel du laboratoire de BotaniqueBiodiversité et de l'Herbier Dakar de l'Université Cheikh Anta Diop de Dakar, la Direction des Parcs Nationaux pour la mise en disposition du parc, les populations des villages périphériques du parc et Société d'Aménagement et d'Exploitation des terres du Delta (SAED) antenne Richard toll qui ont facilité la collecte des données.

\section{REFERENCES}

Bissoue AN, Enjoh N, Ndjouondo GP, Dibong SD. 2017. Socio-economic aspect of battle against invasive aquatic plants of nyong river in mbalmayo district. International Journal of Innovation and Applied Studies, 19(2): 363-375.

DOI: http://docplayer.fr/64283937.

Cogels FX. 1997. Suivi et gestion de l'hydrologie et de la qualité des eaux du lac de Guiers (Sénégal). International Association of Hydrological Science, 243: 277-286.

Cottaz C. 2016. Lignes directrices pour l'évaluation des impacts des espèces exotiques envahissantes et leur hiérarchisation. Guideline. no.3.

Dibong SD, Ndjouondo GP. 2014. Inventaire floristique et écologie des algues des rivières Kambo et Longmayagui de la zone humide de Douala (Cameroun). Int. J. Biol. Chem. Sci., 8(6): 2560-2577.

Diouf N, Mbaye MS, Gueye M, Dieng B, Bassene C. 2019. La flore adventice des cultures cotonnières dans le Sénégal Oriental et en Haute Casamance. Int. J. Biol. Chem. Sci., 13(3): 1720-1736.

Donlan CJ, Wilcox C. 2008. Diversity, invasive species and extinctions in insular ecosystems. Journal of Applied Ecology, 45: 1114-1123. DOI: 10.1111/j.1365-2664.2008.01482.x.

Fall O, Fall I, Hori N. 2004. Assessment of the abundance and distribution of the aquatic plants and their impacts in the Senegal river delta: the case of Khouma 
and Djoudj streams. Weed Technology., 18: 1203-1209.

Fall O, Hori N, Kan H, Diop M. 2003. Toward an integrated management plan of the Djoudj Park water resources: Senegal River mouth. Environmental Management, 31(1):14-28.

Gardner RC, Barchiesi S, Beltrame C, Finlayson CM, Galewski T, Harrison I, Paganini M, Perennou C, Pritchard DE, Rosenqvist A, Walpole M. 2015. State of the World's Wetlands and their Services to People: A compilation of recent analyses. Ramsar Briefing Note No. 7. Gland, Switzerland: Ramsar Convention Secretariat, 21p.

Gbocho YF, Diakité RN, Akotto OF, Kouakou E. 2015. Dynamique des populations de mollusques hôtes intermédiaires deSchistosoma haematobium et Schistosoma mansoni dans le lac du barrage de Taabo (sud Côte d'Ivoire). Journal of Animal \& Plant Sciences, 25(3): 3939-3953.

GISD. 2006. Ecology of Ceratophyllum demersum. Global Invasive Species Database.

http://www.iucngisd.org/gisd/species.ph $\mathrm{p}$ ? $\mathrm{sc}=281$.

Gueye M, Noba K. 2016. Etude pédologique et phytosociologique de deux mares dans le Parc National du Niokolo Koba dans un contexte d'invasion biologique par Mimosa pigra. Int. J. Biol. Chem. Sci., 9(6): 2871-2888.

Gueye M. 2009. Etude systématique des cyanophytes et micro algues du parc national des oiseaux du Djoudj (Sénégal). Mémoire de DEA, Université Cheikh Anta Diop, Dakar, p. 70.

Gueye M, Cisse MT, Ndoye F, Noba K. 2016. Dynamique invasive de Mimosa pigra dans un contexte de variabilité du regime hydrologique au Sénégal. International Journal of Innovation and Applied Studies, 16 (4): 681-696.

Kettunen M, Genovesi P, Gollasch S, Pagad S, Starfinger U. 2008. Assessment of the impacts of IAS in Europe and the EU. Institute for European Environmental Policy (IEEP), 44.
Mack RN, Simberloff D, Lonsdale WM, Evans H, Clout M, Bazzaz FA. 2000. Biotic Invasions: Causes, Epidemiology, Global Consequences, and Control. Ecological Applications, 10 (3): 689710.

Mbaye MS. 2015. Etude de référence sur la situation des plantes aquatiques au niveau des axes hydrauliques de la délégation de Dagana de la SAED. Rapport final, 143p.

Michon G. 2015. Contrôle du myriophylle à épi (Myriophyllum spicatum) par l'utilisation de toiles de jute. Rapport final présenté au Ministère des Forêts, de la Faune et des Parcs. Agence de bassinversant des 7, 72.

Ndione RA. 2018. Etude de l'influence de certains paramètre environnementaux sur la dynamique des populations de mollusques hôtes intermédiaires des schistosomes dans la vallée du fleuve Sénégal: exemple d'une méthodes de lutte biologique avec la crevette d'eau douce le Macrobrachium volenhovenii. Communication scientifique, Université Cheikh Anta Diop, Dakar.

Ndour S. 2015. Biologie, ecologie et dynamique d'invasion de deux especes envahissantes dans la vallee du fleuve senegal : Ceratophyllum demersum L. et Najas marina L. Mémoire de Master, Université Cheikh Anta Diop, Dakar, p. 58.

NIWA. 2005. National Institute of Water and Atmospheric Research, New Zealand.

Noba K, Mbaye MS, Coundoul M, Kane A, Hane PD, Ba N, Mbaye N, Guissé A, Faye MN, Tidiane A. 2010. La flore du Parc national des oiseaux de Djoudj, une zone humide du Sénégal. Sécheresse, 21(1): 71-78.

Pimentel D, Mcnair S, Janecka J, Wightman J, Simmonds C, Wong E, Russel L, Zern J, Aquino T, Tsomondo T. 2001. Economic and environmental threats of alien plant, animal, and microbe invasions. Agriculture, Ecosystems and Environment, 84: 1-20.

Pomerleau G. 2017. Plans stratégiques d'intervention pour la gestion des 
espèces exotiques envahissantes identifiées prioritaires dans la zone périphérique du parc national du MontOrford. Essai de Maitrise, Université de Sherbrooke. p. 109.

Priso RJ, Olivier OG, Ndongo D. 2012. Utilisation des macrophytes comme descripteurs de la qualité des eaux de la rivière Kondi dans la ville de Douala (Cameroun-Afrique Centrale). J. Appl. Biosci., 53: 3797-3811.

Poda JN, Wango SP, Sorgho H, Dianou D. 2004. Évolution récente des schistosomoses dans le complexe hydroagricole du Sourou au Burkina Faso. Bull. Soc. Pathol. Exot., 97(1): 1518.

UICN. 2000. Lignes directrices de l'UICN pour la prévention de la perte de diversité biologique causée par des espèces exotiques envahissantes, 25p.

Sarr A, Kinzelbach R, Diouf M. 2011. Diversité spécifique et écologie des mollusques continentaux de la basse vallée du Ferlo (Sénégal). Journal MalaCo., 7: 383-390.

Satin A, Shastry W. 1983. L'Echantillonnage : un Guide non Mathématique ( $2^{\text {nd }}$ edn). Statistique Canada: Ottawa.

Triplet P, Ndiaye S, Diop I. 2014. Plan de gestion du parc national des oiseaux $d u$ Djoudj 2014-2018. Direction des Parcs Nationaux. Ministère de l'Environnement et du Développement Durable, 61p.

Vári Á. 2012. Propagation and growth of submerged macrophytes in Lake Balaton. PhD thesis, Eötvös Loránd University, Budapest, p. 125.

Zongo D, Bagayan M, Tiendrébeogo S, Drabo F, Ouedraogo H, Savadogo B, Bamba I, Yago-Vienne F, Zhang Y, Poda JN. 2017. Assessment of schistosomiasis and intestinal helminths following mass drug administration in the Centre and Plateau Central regions of Burkina Faso. Int. J. Biol. Chem. Sci., 10(4): 1525-1533. 